\title{
Dynamin 2 Inhibitors as Novel Therapeutic Agents Against Cervical Cancer Cells
}

\author{
YOO-YOUNG LEE ${ }^{1 *}$, HYE-KYUNG JEON ${ }^{2 *}$, JUNGEUN LEE ${ }^{3 *}$, JI EUN HONG ${ }^{2}$, IN-GU DO ${ }^{4}$, CHEL HUN CHOI $^{2}$, \\ TAE-JOONG KIM ${ }^{2}$, BYOUNG-GIE KIM ${ }^{2}$, DUK-SOO BAE ${ }^{2}$, YONG-CHUL KIM ${ }^{3}$ and JEONG-WON LEE ${ }^{4}$ \\ ${ }^{I}$ Division of Gynecologic Oncology, Princess Margaret Cancer Centre, \\ University Health Network, University of Toronto, Toronto, ON, Canada; \\ ${ }^{2}$ Department of Obstetrics and Gynecology, Samsung Medical Center, \\ Sungkyunkwan University School of Medicine, Seoul, Republic of Korea; \\ ${ }^{3}$ School of Life Sciences, Gwangju Institute of Science and Technology, Gwangju, Republic of Korea; \\ ${ }^{4}$ Department of Pathology, Kangbuk Samsung Hospital, \\ Sungkyunkwan University School of Medicine, Seoul, Republic of Korea
}

\begin{abstract}
Aim: We investigated the feasibility of dynamin 2 as a potential treatment target in cervical cancer cells. Materials and Methods: We performed tissue microarray for dynamin 2 expression in 208 patients with early cervical cancer and in vitro in HeLa cells with dynamin 2 inhibitors MiTMAB, OcTMAB, Dynasore, and DD-6. Results: Tumor size greater than $2 \mathrm{~cm}$ or tumor invasion of more than half of the entire cervix was associated with expression of dynamin 2 compared to no expression $(p=0.013$, and $p=0.045$, respectively). All dynamin 2 inhibitors significantly reduced proliferation, increased apoptotic activity, and reduced matrix metallopeptidase 9 expression in HeLa cells. Dynasore and DD-6 reduced migration of HeLa cells on laminin 1-coated plates and DD-6 most strongly reduced migration performance on fibronectin-coated plates. Conclusion: Targeting dynamin 2 may be a promising new approach for the treatment of cervical cancer.
\end{abstract}

Cervical carcinoma is still prevalent on a global scale and advanced, metastatic, or recurrent disease is difficult to cure using standard treatment (1). Increasing efforts have been made to identify genomic and proteomic alterations associated with cervical cancer progression and previous

*These Authors equally contributed to this study.

Correspondence to: Associate Professor Jeong-Won Lee, MD, Ph.D., Department of Obstetrics and Gynecology, Samsung Medical Center, Sungkyunkwan University School of Medicine, 81 Irwonro, Gangnam-gu, Seoul, 06351, Korea. Tel: +82 234101382, Fax: +82 234100630, e-mail: garden.lee@samsung.com

Key Words: Cervical cancer, dynamin 2, MiTMAB, OcTMAB, Dynasore, DD-6. studies showed that dynamin 2, a member of the large GTPase superfamily, was differentially expressed between normal cervical epithelium, cervical intraepithelial neoplasm, and invasive cervical cancer tissues and had a prognostic role for predicting recurrence (2-4).

In vitro studies of various types of cancer cells have shown that dynamin 2 is required for extracellular matrix degradation by invasive cancer cells (5) and is also necessary for the endocytosis of several proteins associated with cancer motility and invasiveness (6). Furthermore, it was reported that dynamin 2 plays an important role in vascular endothelial growth factor (VEGF)-mediated angiogenesis (7). This evidence strongly suggests that dynamin 2 is widely involved in tumor progression and metastasis and might be a good candidate target for new treatments that have not been extensively studied in cervical cancer cells.

In this study, we investigated the feasibility of dynamin 2 as a potential treatment target in cervical cancer cells using different dynamin 2 inhibitors.

\section{Materials and Methods}

Patients. With Institutional Review Board approval (2009-09-002002), we searched electronic medical records of patients with primary early cervical cancer (International Federation of Gynecology and Obstetrics, FIGO stage I-II) who were treated with surgery and registered at the Samsung Medical Center of Sungkyunkwan University School of Medicine in Seoul, Korea, between January 2002 and September 2008. A total of 208 patients were selected as candidates for tissue microarray (TMA).

TMA construction and immunohistochemical staining. TMAs were constructed using Beecher Manual Tissue Microarrayer (MTA-1; Beecher Instruments Inc., WI, USA). Four representative tumor regions were taken from donor formalin-fixed paraffin-embedded blocks using a 0.6-mm core punch and arrayed into recipient blocks. 
Immunohistochemical studies were carried out on 4- $\mu$ m TMA sections with primary mouse monoclonal antibody specific for dynamin 2 (BD Biosciences, San Jose, CA, USA). Tissue sections were deparaffinized three times in xylene for a total of $15 \mathrm{~min}$ and subsequently rehydrated. Antigen retrieval was carried out at $97^{\circ} \mathrm{C}$, using PTLink (DAKO, Glostrup, Denmark) for $20 \mathrm{~min}$ in citrate buffer. After blocking endogenous peroxidase activity with $3 \%$ hydrogen peroxidase for $10 \mathrm{~min}$, primary antibody incubation was carried out at a 1:200 dilution for $60 \mathrm{~min}$ at room temperature. The antigen-antibody reaction was detected using the DAKO REAL ${ }^{\mathrm{TM}}$ Envision $^{\mathrm{TM}}$ Detection system, Peroxidase/DAB K5007 (DAKO). Counterstaining was performed with Mayer's hematoxylin. Negative controls (substitution of primary antibody for Tris-buffered saline) were run simultaneously. The slides were assessed by a pathologist (Dr. I-G Do) without knowledge of the clinical outcome.

Interpretation of TMA. Staining for dynamin 2 was considered to be positive when tumor cells showed membranous/cytoplasmic reactivity. Immunohistochemical staining was evaluated by a semiquantitative scoring system with two categories: intensity and percentage of positively stained cells. The intensity was graded on a semiquantitative scale from 0 to 3 , where $0=$ no staining, $1=$ weak staining, $2=$ moderate staining, and $3=$ strong staining. The percentage of positive cells was stratified from 0 to 3 , where no staining in all four TMA cores $=0$, positivity in 1 out of 4 cores $=1$, positivity in 2 out of 4 cores $=2$, and positivity in more than 2 cores $=3$. The two grades were multiplied and the immunohistochemical staining score was determined: 0 as negative, 1 and 2 as weak (score 1), 3 and 4 as moderate (score 2), and 6 and 9 as strongly positive (score 3 ).

Cell lines. Human cervical cancer cell lines HeLa, SiHa, and Ca Ski were obtained from the Korean Cell Line Bank (KCMB, Seoul, South Korea); C-33A was obtained from the American Type Culture Collection (Manassas, VA, USA). Cells were maintained in Dulbecco's modified Eagle's medium (DMEM) (HeLa), minimal essential medium (SiHa, C-33A, MS751), McCoy's 5A (HT-3, ME180), or RPMI-1640 (Ca Ski) medium supplemented with $10 \%$ fetal bovine serum (FBS) and $0.1 \%$ gentamicin sulfate (Gemini Bioproducts, Calabasas, $\mathrm{CA}, \mathrm{USA}$ ) in $5 \% \mathrm{CO}_{2}$ at $37^{\circ} \mathrm{C}$.

Reagents. Cell permeable dynamin inhibitors, 3-hydroxynaphthalene2-carboxylic acid (3, 4-dihydroxybenzylidene) hydrazide (Dynasore), tetradecyltrimethylammonium bromide (MiTMAB) and octadecyltrimethylammonium bromide (OcTMAB) were purchased from Abcam Biochemicals (Cambridge, UK). DD-6 was obtained from Dr. Yong-Chul Kim (School of Life Sciences, Gwangju Institute of Science and Technology, Gwangju, South Korea) and Dr. Sunghoe Chang (Department of Physiology and Biomedical Sciences, Seoul National University College of Medicine, Seoul, South Korea) (8). Dynasore, OcTMAB, and DD-6 were dissolved in dimethyl sulfoxide (DMSO). MiTMAB was dissolved in distilled water.

Small interfering RNA (siRNA) transfection. Dynamin 2 siRNA and negative control siRNA were purchased from Santa Cruz Biotechnology Incorporated (Santa Cruz, CA, USA). Cells were seeded at $3 \times 10^{3}$ cells/well in a 96-well microplate and at $2 \times 10^{5}$ cells/well in 6-well plates in DMEM with $10 \%$ FBS. All siRNAs were transfected into the cells using Lipofectamine 2000 (Invitrogen, San Diego, CA, USA) according to the manufacturer's protocol.
Fluorescence-activated cell sorting (FACS) analysis. At $48 \mathrm{~h}$ after transfection with dynamin 2 siRNA, the relative percentage of apoptotic cells was assessed using the Annexin V-FITC Apoptosis Detection Kit-1 (BD Pharmingen, San Diego, CA, USA) according to the manufacturer's protocol. Briefly, cells were washed twice in PBS and the pellet was resuspended in annexin $\mathrm{V}$ binding buffer at a density of $10^{6}$ cells $/ \mathrm{ml}$. Annexin V fluorescein isothiocyanate (FITC) and propidium iodide (PI) were added ( $1 \mu \mathrm{l}$ of each per $10^{5}$ cells). Samples were gently mixed and incubated for $15 \mathrm{~min}$ at room temperature in the dark before FACS analysis.

3-(4, 5-Dimethylthiazol-2-yl)-2, 5-diphenyl tetrazolium bromide (MTT) assay. For the proliferation assay, HeLa cells were seeded at $3 \times 10^{3}$ cells/well in 96-well microplates, treated with different concentrations of dynamin inhibitors, and incubated at $37^{\circ} \mathrm{C}$ for 24,48 , and $72 \mathrm{~h}$. MTT solution (Amresco, Solon, OH, USA) was then added to the 96well microplates. After incubation for $4 \mathrm{~h}$, the medium was discarded, $100 \mu \mathrm{l}$ of acidic isopropanol $(0.1 \mathrm{~N} \mathrm{HCl}$ in absolute isopropanol) was added, and the plate was shaken gently. Absorbance was measured on an enzyme-linked immunosorbent assay (ELISA) reader at a test wavelength of $540 \mathrm{~nm}$. Each sample was assayed in triplicate.

Western blot analysis. HeLa, SiHa, Ca Ski, and C-33A cells were lysed in PRO-PREP Protein Extraction Solution (Intron Biotechnology, Seongnam, Korea). Protein concentration was determined using a BCA protein assay kit (Thermo, IL, USA). Protein bands were probed with antibodies against dynamin 2 (Santa Cruz Biotechnology), glyceraldehyde 3-phosphate dehydrogenase (GAPDH; Epitomics, Burlingame, USA), or tubulin (Epitomics), and then labeled with horseradish peroxidase-conjugated anti-rabbit antibody (GE Healthcare, Piscataway, NJ, USA). Bands were visualized by enhanced chemiluminescence using an ECL kit (Amersham Biosciences, Buckinghamshire, UK) according to the manufacturer's protocol.

Enzyme-linked immunosorbent assay (ELISA). For analysis of matrix metallopeptidase 9 (MMP9) and active caspase-3 expression, cells were lysed in PRO-PREP Protein Extraction Solution (Intron Biotechnology) after 24-h treatment with dynamin inhibitors. The protein concentration was determined using a BCA protein assay kit (Thermo). ELISA kits were used to measure concentrations of human MMP9 (Abnova, Walnut, CA, USA) and active caspase-3 (Invitrogen) as described by the manufacturer. The samples were measured in triplicate.

Migration assay. HeLa cells were seeded at $1 \times 10^{5}$ cells/well in a 24-well plate and migration was measured using the Radius Cell Migration Assay Kit (Cell Biolabs, San Diego, CA, USA) according to the manufacturer's protocol. The plates were precoated with various extracellular matrix (ECM) proteins (laminin I, fibronectin, collagen I) to monitor the migratory properties of cells. After 17-h treatment with $10 \mu \mathrm{M}$ dynamin inhibitors, cells were observed under a light microscope at $\times 40$ magnification.

Statistical analysis. The Wilcoxon rank sum test or two-sample $t$ test was used to compare the median or mean values, respectively, after checking whether the data had non-normal or normal distributions according to the Shapiro-Wilks test. Statistical analyses were performed using SPSS software (version 18.0; SPSS, Chicago, IL, USA). A $p$-value of less than 0.05 was considered statistically significant and all reported $p$-values are two-sided. 


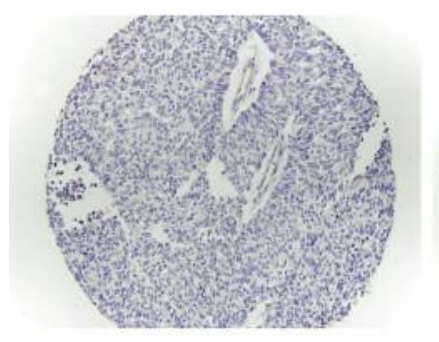

Negative (0)

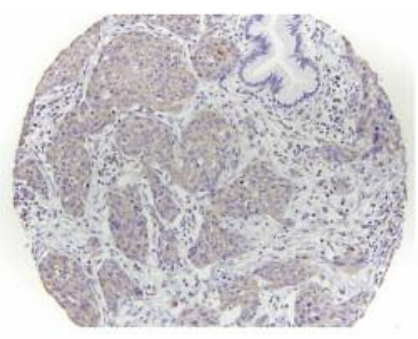

Weak (1)

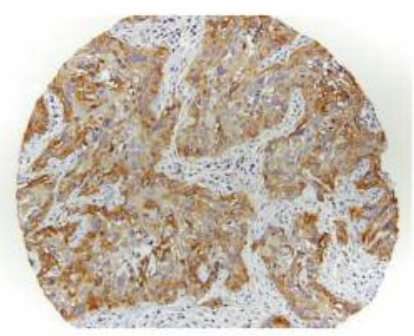

Moderate (2)

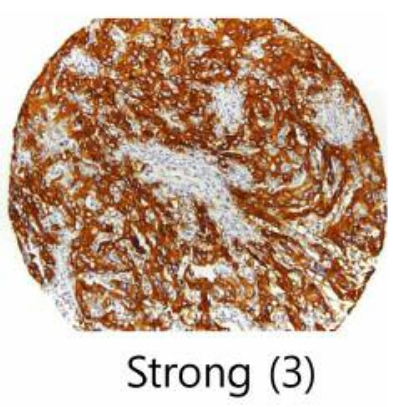

Figure 1. Dynamin 2 expression in human cervical cancer as shown by immunohistochemical staining. Immunohistochemical staining was evaluated by a semi-quantitative scoring system with two categories: intensity and percentage of positively stained cells. The two scores were multiplied and the immunohistochemical staining score was determined: 0 as negative; 1 and 2 as weak (score 1); 3 and 4 as moderate (score 2); and 6 and 9 as strongly positive (score 3).

Table I. Dynamin 2 expression according to clinicopathological factors in cervical cancer tissue.

\begin{tabular}{|c|c|c|c|c|c|c|c|}
\hline & & \multicolumn{4}{|c|}{ Dynamin 2 expression, $\mathrm{n}(\%)$} & \multirow[b]{2}{*}{ Total } & \multirow[b]{2}{*}{$p$-Value } \\
\hline & & 0 & 1 & 2 & 3 & & \\
\hline \multirow[t]{4}{*}{ FIGO stage } & IB1 & $79(54.5)$ & $20(13.8)$ & $27(18.6)$ & $19(13.1)$ & $145(100.0)$ & 0.692 \\
\hline & IB2 & $11(55.0)$ & $3(15.0)$ & $4(20.0)$ & $2(10.0)$ & $20(100.0)$ & \\
\hline & IIA & $17(50.0)$ & $5(14.7)$ & $10(29.4)$ & $2(5.9)$ & $34(100.0)$ & \\
\hline & IIB & $4(44.4)$ & $1(11.1)$ & $4(44.4)$ & 0 & $9(100.0)$ & \\
\hline \multirow[t]{2}{*}{ Tumor size } & $\leq 2 \mathrm{~cm}$ & $29(70.7)$ & $3(7.3)$ & $7(17.1)$ & $2(4.9)$ & $41(100.0)$ & 0.101 \\
\hline & $>2 \mathrm{~cm}$ & $82(49.1)$ & $26(15.6)$ & $38(22.8)$ & $21(12.6)$ & $167(100.0)$ & $0.013 *$ \\
\hline \multirow[t]{2}{*}{ Depth of invasion } & $\leq 1 / 2$ & $34(65.4)$ & $4(7.7)$ & $6(11.5)$ & $8(15.4)$ & $52(100.0)$ & 0.041 \\
\hline & $>1 / 2$ & 77 (49.4) & $25(16.0)$ & $39(25.0)$ & $15(9.6)$ & $156(100.0)$ & $0.045^{*}$ \\
\hline
\end{tabular}

*Additional comparison between expression and no expression of dynamin 2.

\section{Results}

We enrolled 208 patients with cervical cancer (FIGO stage IB 1 to IIB) to investigate the expression of dynamin 2 in cervical cancer tissue and compared their expression levels according to the pathological findings after surgery. Different expression levels of dynamin 2 were observed in the cervical cancer samples, as shown in Figure 1. Tumor size greater than $2 \mathrm{~cm}$ or tumor invasion of more than half of the entire cervix was associated with positive expression of dynamin 2 compared to no expression (scores of 1,2 , and $3 \mathrm{vs}$. 0 , $p=0.013$ and $p=0.045$, respectively, Table I).

Cervical cancer cell lines also exhibited different expression levels of dynamin 2 , and the strong expression of dynamin 2 in HeLa cells was markedly reduced by transfection with specific siRNA as shown in Figure 2A. Dynamin 2-specific siRNA reduced cell proliferation and induced apoptosis in a dose-dependent manner (Figure 2B and $\mathrm{C}$ ). Based on these findings, further experiments with dynamin 2 inhibitors were performed in HeLa cells.

Different dynamin 2 inhibitors, MiTMAB, OcTMAB, Dynasore and DD-6, were used to inhibit dynamin 2 activity in HeLa cells and all of these inhibitors significantly reduced cell proliferation as assessed by MTT assay (Figure 3A). Specifically, inhibition of proliferation was dose-dependent with MiTMAB and Dynasore at 48 and $72 \mathrm{~h}$. All of the inhibitors also markedly increased apoptotic activity in $\mathrm{HeLa}$ cells (Figure 3B).

Finally, we investigated the effect of dynamin 2 inhibitors on MMP9 expression and cell migration in HeLa cells, as shown in Figure 4. Different effects were observed for different inhibitors. For example, the expression of MMP9 was significantly reduced by treatment with OcTMAB, Dynasore, and DD-6. In the migration assay, Dynasore and DD-6 reduced cell migration and DD-6 most strongly reduced migration in fibronectin-coated wells.

\section{Discussion}

Dynamin 2, the most ubiquitous isoform of the dynamin family, has broad physiological functions in the regulation of vesicle transport and endocytosis trafficking $(9,10)$. Human papilloma virus (HPV) can enter the cytoplasm of cervical epithelium cells via dynamin 2-dependent 

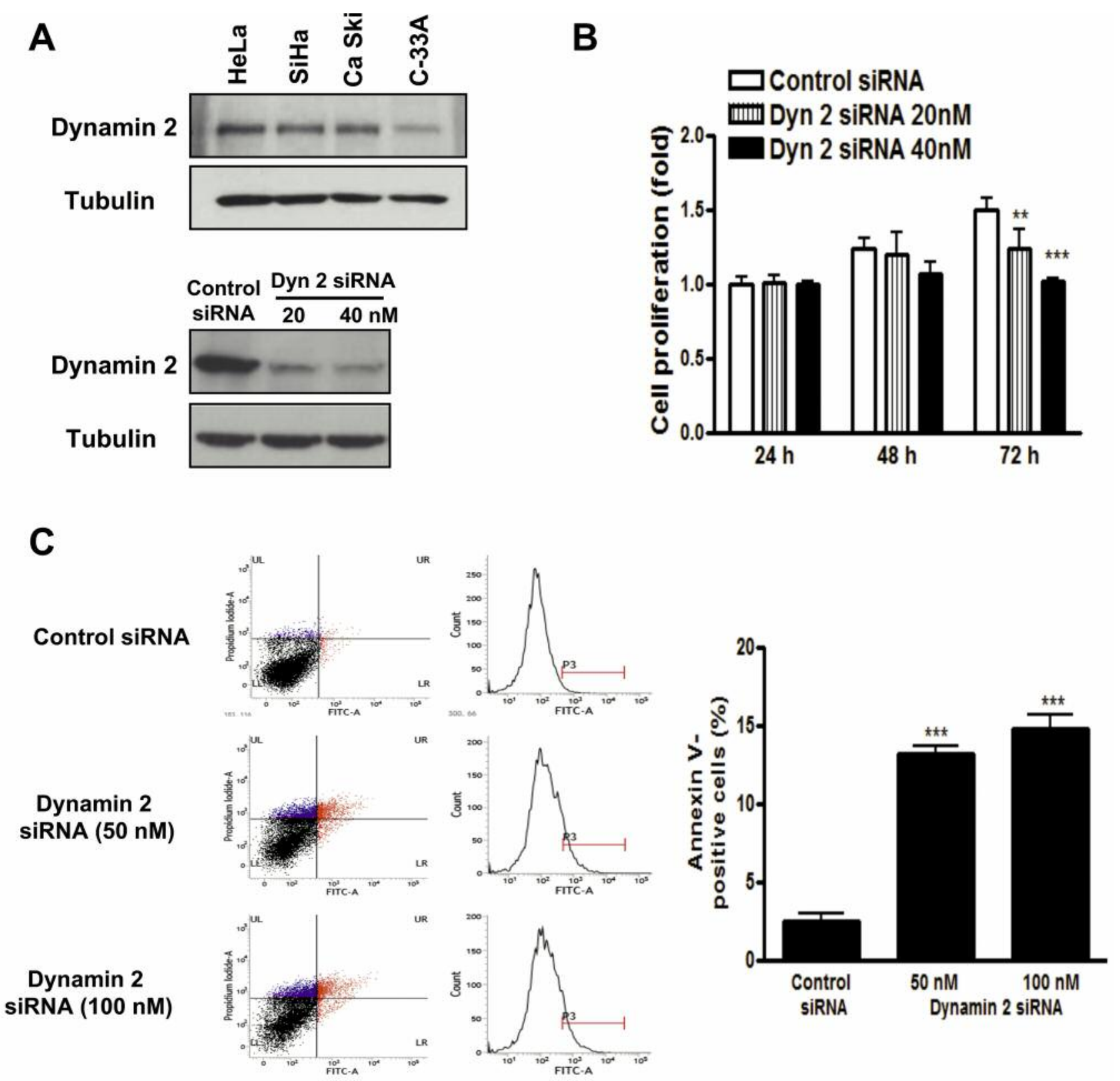

Figure 2. Effects of dynamin 2-specific siRNA on cell survival and apoptosis in HeLa cells. Dynamin 2 was highly expressed in HeLa cells and its expression was reduced by dynamin 2-specific siRNA (A). Cell proliferation (B) was markedly reduced and apoptosis $(C)$ was significantly increased by transfection with dynamin 2 -specific siRNA. *p<0.05, **p<0.01 and $* * * p<0.001$.

endocytosis $(11,12)$, and this may eventually lead to progression to cervical intraepithelial neoplasm and invasive cervical cancer. Interestingly, dynamin 2 was expressed at different levels with disease progression in our previous studies $(2,3)$.

Our current results showed that inhibition of dynamin 2 resulted in a significant increase in apoptosis and decrease in cell proliferation and migration of cervical cancer cells.
Similar findings were also reported in various cancer cells derived from colon, breast, lung, pancreas, brain, and prostate (13-15). Most of these studies reported that dynamin 2 inhibition is not only associated with prevention of rapid turnover of focal adhesion molecules, which are required for cell migration, but is also associated with the arrest of cell kinesis, resulting in decreased cell proliferation and increased apoptosis. For example, knockdown of 

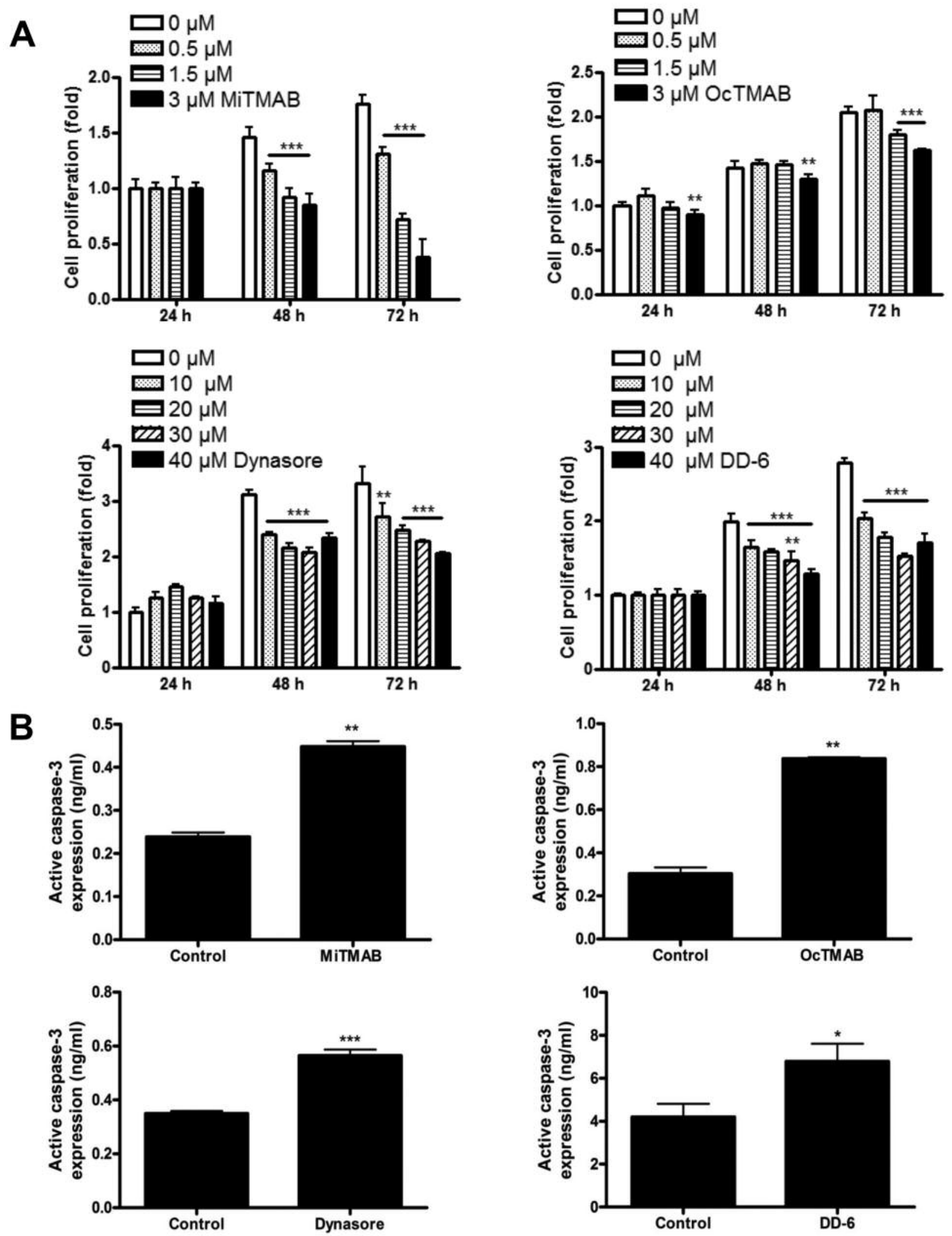

Figure 3. Effect of dynamin 2 inhibitors on proliferation and apoptosis of HeLa cells. Cell proliferation (A) was significantly reduced and apoptosis (B) was significantly increased by treatment with different inhibitors of dynamin 2 (MiTMAB, OcTMAB, Dynasore, DD-6). *p<0.05, **p<0.01 and ***p<0.001. 


\section{A}
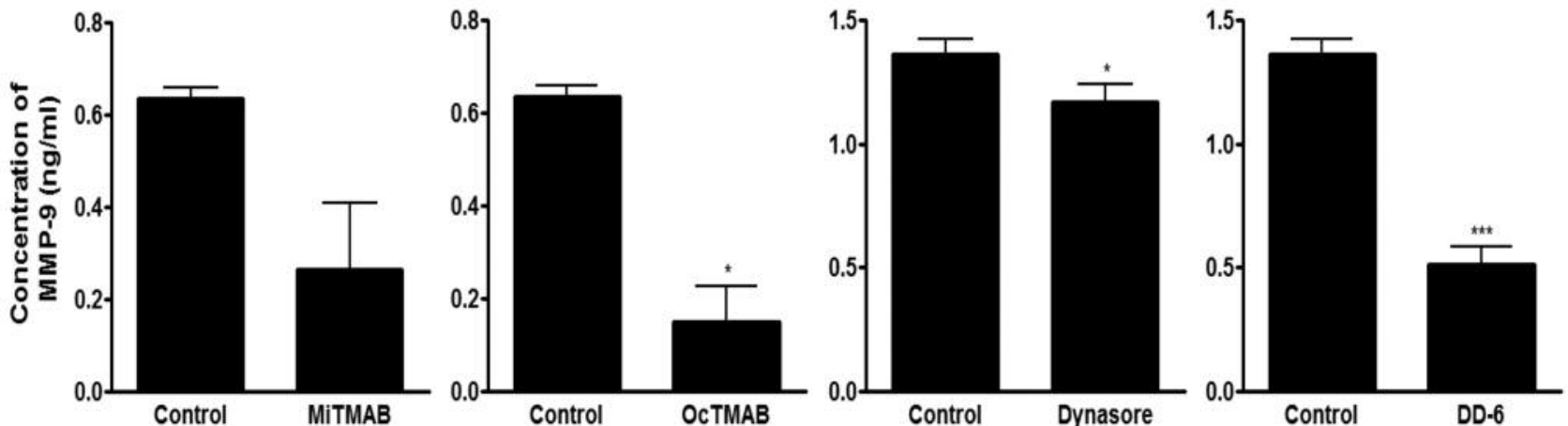

B

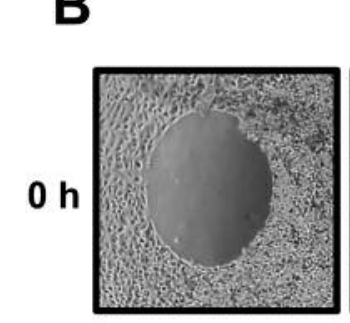

Uncoated
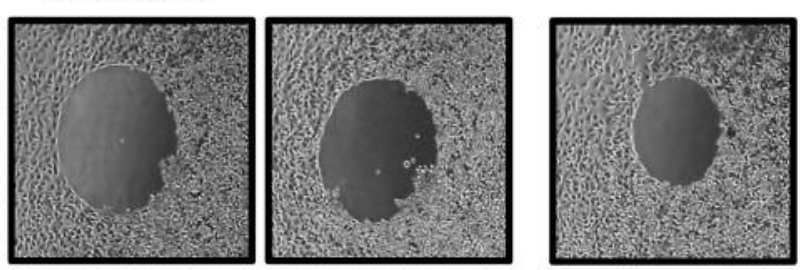

Laminin I
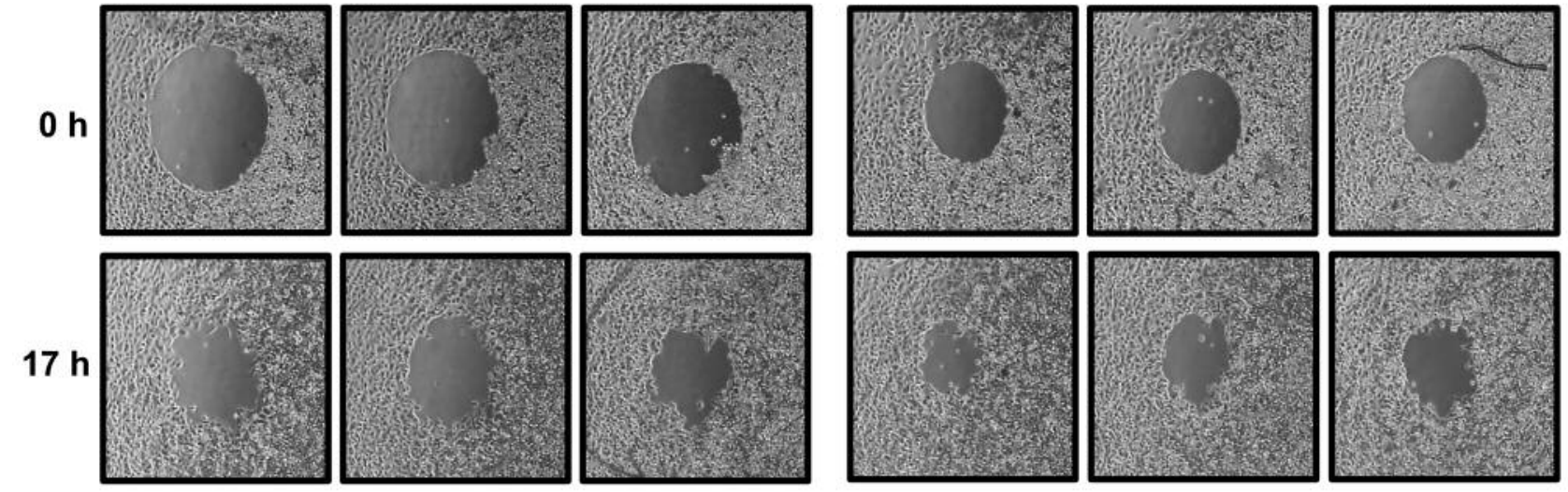

Fibronectin

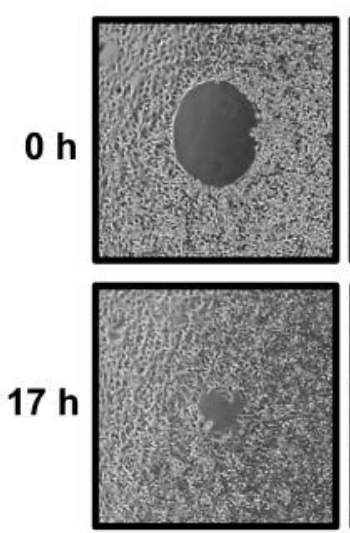

Control
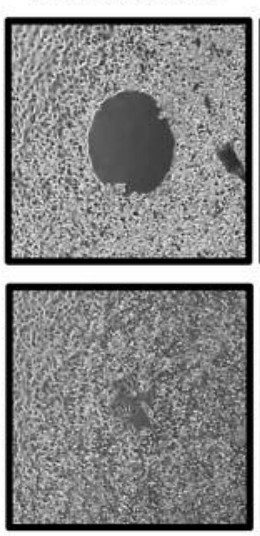

Dynasore

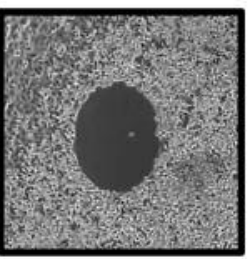

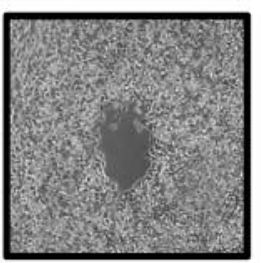

DD-6
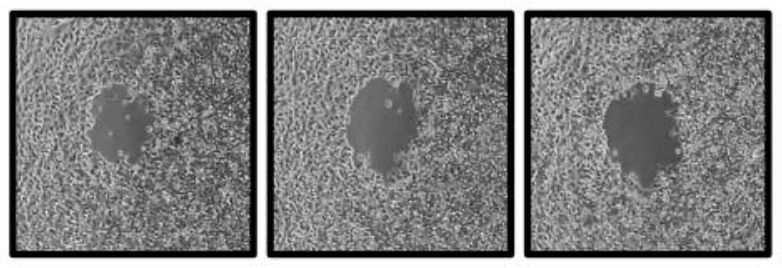

\section{Collagen I}
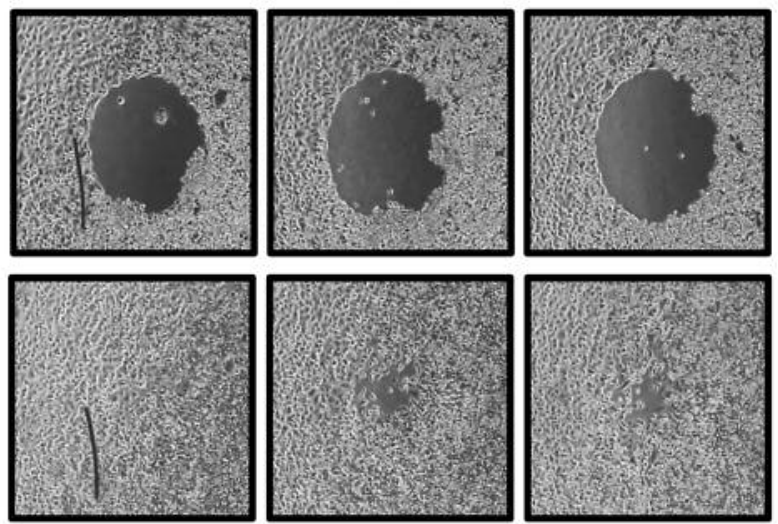

Control

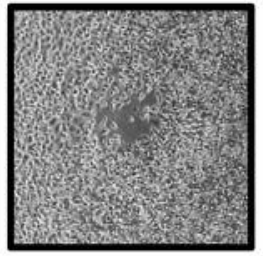

Dynasore

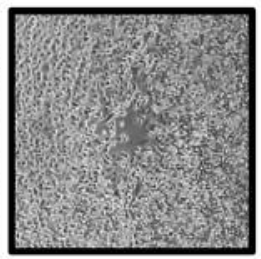

DD-6

Figure 4. Effect of dynamin 2 inhibitors on matrix metallopeptidase (MMP) 9 expression (A) and migration (B) of HeLa cells. Expression of MMP9 was significantly reduced by different dynamin 2 inhibitors. Dynasore and DD-6 reduced the migration performance of HeLa cells on plates coated with laminin I, fibronectin, or collagen I. * $p<0.05$, **p<0.01 and ***p<0.001. 
dynamin 2 blocks beta 1 integrin internalization, leading to impaired focal adhesion disassembly and cell migration (6), and silencing of dynamin 2 induces cell death specifically following cytokinesis failure via the intrinsic apoptotic pathway involving cleavage of caspase-8, -9, and -3 (14). Furthermore, higher expression of dynamin 2 in human cancer tissue was associated with higher cancer-specific mortality (13). The results of our study suggest that dynamin 2 expression in cervical cancer tissue was associated with larger tumor size and increased local invasion. However, we did not find any prognostic difference in patients with cervical cancer based on the expression level of dynamin 2. This should be clarified in future studies but considering that most of our patients had early-stage cervical cancer, which usually has good survival outcomes, our study may have had insufficient power to detect a survival difference.

Studies of various dynamin 2 inhibitors, including Dynasore (16), long chain amines and ammonium salts (MiTMABs, OcTMAB) (14), and dynoles (15), revealed that they block the abscission phase of cytokinesis causing cell death. Here, we test our novel dynamin 2 inhibitor (DD-6), which was reported to more potently inhibit transferrin uptake and dynamin GTPase activity than dynasore (8). Targeting cytokinesis with DD-6 may be a promising new approach for the treatment of cancer.

Another implication of dynamin 2 inhibitors for tumor control is their anti-angiogenesis role through reducing the expression of angiogenic receptors in endothelial cells (17, 18). However, the molecular mechanisms by which dynamin 2 contributes to cancer progression are not fully understood. A recent study reported that there are multiple hypoxia-inducible factor (HIF)-binding sites in the promoter domain of dynamin 2 and, interestingly, reciprocal regulation of dynanim 2 and HIF in ovarian cancer cells (19). The authors found that miR-199a encoded by the opposite strand of dynamin 2 reduced HIF expression, cell migration, and metastasis of ovarian cancer cells, which are opposing results from those of previous studies mentioned above. It is not clear which factors are associated with these discrepant results and different tumor biology of each cell type might partially explain this phenomenon (20).

In conclusion, targeting dynamin 2 with various specific inhibitors may be a promising new approach for the treatment of cervical cancer.

\section{Acknowledgements}

This research was supported by a grant of the Korea Health Technology R\&D Project through the Korea Health Industry Development Institute (KHIDI), funded by the Ministry of Health \& Welfare, Republic of Korea (HI14C3418).

\section{References}

1 Hirte H, Kennedy EB, Elit L and Fung Kee Fung M: Systemic therapy for recurrent, persistent, or metastatic cervical cancer: A clinical practice guideline. Curr Oncol 22(3): 211-219, 2015.

2 Lee YY, Song SY, Do IG, Kim TJ, Kim BG, Lee JW and Bae DS: Dynamin 2 expression as a biomarker in grading of cervical intraepithelial neoplasia. Eur J Obstet Gynecol Reprod Biol 164(2): 180-184, 2012.

3 Lee YY, Do IG, Park YA, Choi JJ, Song SY, Kim CJ, Kim MK, Song TJ, Park HS, Choi CH, Kim TJ, Kim BG, Lee JW and Bae DS: Low dynamin 2 expression is associated with tumor invasion and metastasis in invasive squamous cell carcinoma of cervix. Cancer Biol Ther 10(4): 329-335, 2010.

4 Lee YY, Kim TJ, Kim JY, Choi CH, Do IG, Song SY, Sohn I, Jung SH, Bae DS, Lee JW and Kim BG: Genetic profiling to predict recurrence of early cervical cancer. Gynecol Oncol 131(3): 650-654, 2013.

5 Baldassarre M, Pompeo A, Beznoussenko G, Castaldi C, Cortellino S, McNiven MA, Luini A and Buccione R: Dynamin participates in focal extracellular matrix degradation by invasive cells. Mol Biol Cell 14(3): 1074-1084, 2003.

6 Chao WT and Kunz J: Focal adhesion disassembly requires clathrin-dependent endocytosis of integrins. FEBS Lett 583(8): 1337-1343, 2009.

7 Bhattacharya R, Kang-Decker N, Hughes DA, Mukherjee P, Shah V, McNiven MA and Mukhopadhyay D: Regulatory role of dynamin-2 in vegfr- $/ \mathrm{kdr}$-mediated endothelial signaling. FASEB J 19(12): 1692-1694, 2005.

8 Lee S, Jung KY, Park J, Cho JH, Kim YC and Chang S: Synthesis of potent chemical inhibitors of dynamin gtpase. Bioorg Med Chem Lett 20(16): 4858-4864, 2010.

9 Stowell MH, Marks B, Wigge P and McMahon HT: Nucleotidedependent conformational changes in dynamin: Evidence for a mechanochemical molecular spring. Nat Cell Biol 1(1): 27-32, 1999.

10 Praefcke GJ and McMahon HT: The dynamin superfamily: Universal membrane tubulation and fission molecules? Nat Rev Mol Cell Biol 5(2): 133-147, 2004.

11 Schelhaas M, Shah B, Holzer M, Blattmann P, Kuhling L, Day PM, Schiller JT and Helenius A: Entry of human papillomavirus type 16 by actin-dependent, clathrin- and lipid raft-independent endocytosis. PLoS Pathog 8(4): e1002657, 2012.

12 Smith JL, Campos SK and Ozbun MA: Human papillomavirus type 31 uses a caveolin 1- and dynamin 2-mediated entry pathway for infection of human keratinocytes. J Virol 81(18): 9922-9931, 2007.

13 Xu B, Teng LH, Silva SD, Bijian K, Al Bashir S, Jie S, Dolph M, Alaoui-Jamali MA and Bismar TA: The significance of dynamin 2 expression for prostate cancer progression, prognostication, and therapeutic targeting. Cancer Med 3(1): 14-24, 2014.

14 Joshi S, Braithwaite AW, Robinson PJ and Chircop M: Dynamin inhibitors induce caspase-mediated apoptosis following cytokinesis failure in human cancer cells and this is blocked by bcl-2 overexpression. Mol Cancer 10: 78, 2011.

15 Chircop M, Perera S, Mariana A, Lau H, Ma MP, Gilbert J, Jones NC, Gordon CP, Young KA, Morokoff A, Sakoff J, O'Brien TJ, McCluskey A and Robinson PJ: Inhibition of dynamin by dynole 34-2 induces cell death following cytokinesis failure in cancer cells. Mol Cancer Ther 10(9): 1553-1562, 2011. 
16 Thompson HM and McNiven MA: Discovery of a new 'dynasore'. Nat Chem Biol 2(7): 355-356, 2006.

17 Gu C, Yaddanapudi S, Weins A, Osborn T, Reiser J, Pollak M, Hartwig J and Sever S: Direct dynamin-actin interactions regulate the actin cytoskeleton. EMBO J 29(21): 3593-3606, 2010.

18 Otsuka A, Abe T, Watanabe M, Yagisawa H, Takei K and Yamada H: Dynamin 2 is required for actin assembly in phagocytosis in sertoli cells. Biochem Biophys Res Commun 378(3): 478-482, 2009.

19 Joshi HP, Subramanian IV, Schnettler EK, Ghosh G, Rupaimoole R, Evans C, Saluja M, Jing Y, Cristina I, Roy S, Zeng Y, Shah $\mathrm{VH}$, Sood AK and Ramakrishnan S: Dynamin 2 along with microrna-199a reciprocally regulate hypoxia-inducible factors and ovarian cancer metastasis. Proc Natl Acad Sci USA 111(14): 5331-5336, 2014.

20 Natarajan A, Yardimci GG, Sheffield NC, Crawford GE and Ohler U: Predicting cell-type-specific gene expression from regions of open chromatin. Genome Res 22(9): 1711-1722, 2012.

Received September 20, 2016 Revised October 5, 2016 Accepted October 10, 2016 